\title{
Conditioned and unconditioned stress-induced analgesia: Stimulus preexposure and stimulus change
}

\author{
STEVEN F. MAIER and LINDA R. WATKINS \\ University of Colorado, Boulder, Colorado
}

\begin{abstract}
Aversive events such as electric shock lead to analgesia. There have been differing views with regard to whether the aversive event itself can lead to analgesia as a direct unconditioned reaction, or whether the analgesia is instead a reaction to fear conditioned to cues present during shock or to other associative processes initiated by the aversive event. Maier $(1989,1990)$ has argued that aversive events such as shock lead to both types of analgesia, with the type observed depending on test conditions. Unconditioned analgesia was argued to be present soon after shock, with conditioned analgesia replacing the unconditioned form if the subject is allowed to remain in the shock environment. Consistent with this argument, the experiments reported here show that (1) preexposure to the environment in which shock later occurs has no effect on the analgesia soon after shock, but eliminates the later analgesia; (2) the initial postshock analgesia is unaffected by removing the subject from the shock environment to a different environment, but the later reaction is prevented by such a change; (3) returning the subject to the shock environment after confinement in a nonshock environment rearouses an analgesic reaction; and (4) this rearousal does not occur if the subject has first been confined to the shock environment without shock.
\end{abstract}

Exposure to a variety of aversive stimuli under a large number of differing circumstances produces an analgesic reaction as assessed by standard measures of pain sensitivity/ reactivity (Hayes, Bennett, Newlon, \& Mayer, 1978). This phenomenon has most often been referred to as stressinduced analgesia (SIA). Because of the potential theoretical and practical importance of analgesic reactions, considerable effort has been directed at understanding the psychological nature of SIA, as well as the physiological mechanisms responsible. Conflicting evidence has emerged, and differing views have been developed.

The present paper addresses a number of issues concerning the psychological nature of the analgesia. Most research exploring the nature of SIA has used electric shock as the inducing stimulus. In considering the analgesia produced by shock, it is useful to separately consider reactions to small amounts of shock (1-20 shocks that are fairly brief in duration, say $5 \mathrm{sec}$ or less) and large amounts of shock (80 or more shocks in this duration range). There is evidence for at least three different possibilities concerning the psychological nature of the analgesia observed after small amounts of shock: (1) It is an unconditioned reaction (UCR) to the shock itself or to some sensory consequence of the shock (Maier, 1986; Terman, Shavit, Lewis, Cannon, \& Liebeskind, 1984); (2) it is a conditioned response (CR) and cannot be elicited

\footnotetext{
This research was supported by National Science Foundation Grant BNS 88-09527 and National Institute of Mental Health Research Scientist Award MH 00314 . Correspondence concerning this article should be addressed to Steven F. Maier, Department of Psychology, Campus Box 345, University of Colorado, Boulder, CO 80309.
}

directly by shock; fear is rapidly conditioned to contextual cues by shock, and the analgesia is a reaction to conditioned fear (Fanselow, 1984); and (3) it is a reaction to the memory representation of shock (Grau, 1987a, 1987b). Maier (1986) has argued that exposure to inescapable shocks sufficient to produce "learned helplessness effects" elicits a new analgesic process that is neither a UCR to shock nor a reaction to conditioned fear or to representations of shock. Here, the analgesic reaction is argued to be a response to learning that the inescapable shocks are uncontrollable. Once initiated, it runs its course and it masks any conditioned effects that might be present.

There is evidence to support each of these views. Maier (1989) has argued that much of the seeming discrepancy in findings can be resolved by considering a number of experimental variables that have differed among the experiments supporting the various views. These variables are the number of shocks, the time between the last shock and pain testing, and whether the subjects are removed from the environment in which shocks had occurred for the pain testing. Obviously, testing soon after the last shock and removing the subject from the presence of cues that had been paired with shock would favor obtaining evidence for unconditioned effects, whereas testing in the shock environment and allowing larger amounts of time to elapse between shock and testing would favor conditioned effects. Indeed, data supporting the view that the analgesic reaction after small amounts of shock reflects a UCR to shock were obtained either within 2 min of the last shock (Maier, 1989) or with testing after removal of the subject from the shock apparatus (Klein, Lovaas, Terman, \& Liebeskind, 1983; Terman et al., 1984). Data 
supporting purely conditioned effects in the shock environment generally have reflected summed data across a 10min postshock period (e.g., Fanselow, 1984).

In the most critical experiments supporting Maier's argument, rats received either 1,5 , or 80 inescapable tailshocks while restrained in Plexiglas tubes (Maier, 1989). They then received radiant-heat-pain test trials (tail-flick test) $2,4,6,8$, and 10 min after the last shock of the series, while being left totally undisturbed in their Plexiglas tubes. A single shock led to an analgesia that had dissipated by 8 min postshock, whereas 5 and 80 shocks produced an analgesia that persisted for the $10 \mathrm{~min}$ of testing. A separate set of groups received identical treatments, but while anesthetized with pentobarbital (tail flick to radiant heat can be measured in anesthetized animals since it is a spinal reflex). Here, there was a brief analgesia ( 2 min) after 1 shock, an analgesia that persisted 4 min postshock after 5 shocks, and no analgesia after 80 shocks. On the assumption that unconditioned reactions to shock as a sensory input might be present in anesthetized animals but that the development of conditioning and/or memorial representations should not be present, Maier (1989) argued that the data suggested a brief UCR to shock that habituates over the course of $\mathbf{8 0}$ shocks. The opiate antagonist naltrexone administered before the shock series blocked the brief analgesia after 1 shock, but not the analgesia after 5 shocks. Thus, the UCR was opioid or nonopioid in nature, depending on the number of shocks. This brief unconditioned analgesia after 1 and 5 shocks was then replaced by a conditioned analgesia if the rat was awake. This second analgesia occurring 4-10 min after the last shock in the 1- and 5-shock conditions was blocked by naltrexone, indicating that its production required an opiate synapse (Watkins, Cobelli, \& Mayer, 1982). Finally, the entire analgesia after 80 inescapable shocks in the awake animal was blocked by naltrexone.

The argument was thus that shock produces an immediate analgesic UCR that can be either opioid or nonopioid, that this UCR is replaced by a second analgesia that is a CR and is opioid under the conditions used, and that after 80 shocks an opioid analgesia is produced that is a reaction to the learning of uncontrollability and that is neither a UCR to shock nor a CR in any traditional sense. To further test these ideas, Maier (1989) gave rats either 1,5 , or 80 shocks as before, but the animals were immediately removed from the shock tubes after their last shock and placed in different tubes in a different room for tail-flick testing. The rationale was that now any analgesia that was a CR to cues present during shock should not occur, although UCRs and reactions initiated by learning about uncontrollability might be expected to persist in the new tubes. The results were that in the new environment, 1 and 5 shocks were followed by only the initial brief analgesia that was opioid after 1 shock and nonopioid after 5 shocks. The opioid analgesia 4-10 min after the last shock did not occur. In contrast, the analgesia after 80 shocks still occurred in the new tubes across the entire 10 min of testing and was still reversed by naltrexone.

Although these data are consistent with the view that the analgesia soon after 1 or 5 shocks is a UCR to shock, that the analgesia at later timepoints is a CR, and that the analgesia after 80 shocks is neither, there are alternative possibilities. The analgesia that occurs up to $4 \mathrm{~min}$ postshock and the analgesia that occurs later are clearly different, but one does not have to be a UCR and the other a CR. It is not necessary to grant that a reaction's presence during the anesthetized state requires that the reaction be a UCR, nor is it necessary that nonoccurrence of a reaction after removal from the shock tubes means that the reaction is a CR. For example, removing the subject and placing it in a new environment for pain testing not only removes cues that had been paired with shock, but also provides potentially disruptive stimulation. Perhaps the second analgesia is a nonassociative consequence of the first analgesia that is easily disrupted by new cues and requires circuitry affected by pentobarbital. There is really nothing in the Maier (1989) experiments to prove that the analgesia 4-10 min postshock is a CR. Nor do the data reported by Maier (1989) conclusively prove that the analgesia after 80 shocks is not a CR. It could be a CR that is so potent that removing the animal from the shock cues fails to disrupt it.

The purpose of the present experiments was to further explore these issues.

\section{EXPERIMENT 1A}

Traditional control procedures used to determine the associative nature of a reaction are not useful in the present circumstance. This is because the question posed is not whether analgesia can be associatively conditioned to environmental cues-it surely can be conditioned (e.g., MacLennan, Jackson, \& Maier, 1980). Rather, the questions are whether (1) the analgesia 2-4 min after a single session of 1 and 5 tailshocks delivered to rats restrained in tubes is unconditioned, (2) the analgesia 4-10 min after the last tailshock is a conditioned reaction elicited by the cues present, and (3) the entire reaction following 80 tailshocks is a reaction that is not associatively elicited by the cues present. An examination, for example, of whether analgesia occurs in the tubes if the tubes are paired with shock but does not occur if the tubes are unpaired with shock would answer whether analgesia is conditionable, but would not speak to whether the hypoalgesias of interest here are or are not associatively triggered.

A different approach is to employ manipulations that are known to modify the strength of conditioning and that can be applied before a single session of tailshock. If the arguments made concerning analgesia are correct, such manipulations should have no effect on the analgesia 2-4 min after 1 and 5 shocks, or on any reaction after 80 shocks across the $10 \mathrm{~min}$ of testing. The analgesia 4-10 min after 1 and 5 shocks should be altered. Pre- 
exposure to the environment in which the shocks are delivered is an obvious choice. Preexposure to the cues that will later be paired with the unconditioned stimulus (UCS) is well known to reduce conditioning to those cues, a phenomenon called "latent inhibition" (Lubow and Moore, 1959).

In Experiment 1, rats were exposed to either the environment in which they later received shock or to a different environment. Controls were exposed to a different environment rather than remaining untreated in order to control for handling, restraint, and so forth. The animals then received either 1,5 , or 80 inescapable tailshocks, followed by tail-flick-to-radiant-heat tests $2,4,6,8$, and $10 \mathrm{~min}$ postshock. The shock treatments were preceded by naltrexone or saline to ensure that the preexposures did not alter the nature of any analgesia that might have occurred.

\section{Method}

Subjects. The subjects were 96 male Sprague-Dawley rats, 80-90 days old at the time of testing. The rats were individually housed and maintained on a 12:12-h light:dark cycle, with testing typically occurring in the first few hours of the light part of the cycle.

Apparatus. Inescapable shocks were administered while the rats were restrained in Plexiglas tubes $17.5 \mathrm{~cm}$ in length and $7.0 \mathrm{~cm}$ in diameter. The animal's tail extended from the rear of the tube through a notch cut in the middle of the rear door. This design allowed tail-flick pain testing without removing the subject from the apparatus. Unscrambled $1.0 \mathrm{~mA}$ shocks were delivered by shock sources modeled after the Grason-Stadler Model 700 shock source through electrodes taped to the rat's tail and augmented by electrode paste. The electrodes were constructed from fuse clips modified to fit onto the tail. The restraining tubes sat in plastic, insulated, sound-attenuating chambers that were illuminated by a single 28-V lamp. Masking noise was present and was $80 \mathrm{~dB}$ in intensity.

Pain testing was conducted using a tail-flick apparatus consisting of a $43.0 \times 17.7 \times 8.0 \mathrm{~cm}$ metal box that supported a $7.4 \times 3.0 \mathrm{~cm}$ aluminum plate. A shallow groove was cut in this plate, into which the rat's tail was placed during a test trial. A General Electric $150-\mathrm{W}$ projector spotlight was mounted above the slot. A condenser lens was located between the light source and the rat's tail and focused the light beam. A lateral deflection of the tail of 5-7 mm activated a photocell receiver and automatically terminated the test trial. The heat was adjusted so that naive control animals showed tail-flick latencies in the 4-sec range.

A second room was available in which rats were never shocked. When restrainers were put in this room, there was no light at all and no masking noise.

Procedure. All rats were first accustomed to handling. They then received four daily 30 -min placements into Plexiglas restraining tubes. For half the animals, the tubes were located in the environment in which shock would later occur (the room in which the animals are routinely shocked, with $28-\mathrm{V}$ light and masking noise present), and for the other half, the tubes were located in the room in which shock never occurred, with no light or masking noise. On Day 5, half of the animals in each preexposure condition received naltrexone ( $7 \mathrm{mg} / \mathrm{kg} \mathrm{s.c.)}$ and half received saline. Twenty minutes later, the animals were all placed in tubes that were placed in the shock environment (light and masking noise present). The rats were given $5 \mathrm{~min}$ to adapt and then received three baseline tail-flick tests. A cutoff of $8.0 \mathrm{sec}$ was employed. That is, if a tail flick had not occurred after $8 \mathrm{sec}$, the heat was terminated and an 8.0-sec latency recorded. The last two tests were averaged together to form the baseline score. One third of each of the subgroups then received I tailshock $5 \mathrm{sec}$ in duration and $1.0 \mathrm{~mA}$ in intensity; one third received 5 such shocks at an average intershock interval of $1 \mathrm{~min}$; and one third received 80 such shocks. Tail-flick tests were conducted $2,4,6,8$, and 10 min after the last of the shocks. The animals remained in the shock tubes and in the shock enclosures during the entire duration of testing. Thus, the design was a $2 \times 2 \times 3$ factorial. with preexposure in the shock environment or a different environment, naltrexone or saline, and 1, 5, or 80 shocks as factors, with five repeated tests occurring after the last shock without removing the subjects from the tubes.

\section{Results and Discussion}

The data from this experiment could be analyzed as a $2 \times 2 \times 3 \times 5$ factorial with repeated measures. However, the complexity of interpreting 3- and 4-way interactions suggests a simpler approach. For ease of presentation and clarity of interpretation, the data will be shown and analyzed separately for the 1-, 5-, and 80-shock conditions. This choice is made because there is no theoretical reason to compare the amount of analgesia produced by each of these shock conditions. The theoretical interest in the experiment is in determining the effects of preexposure, naltrexone, and time of testing following each of these shock conditions.

Figure I shows tail-flick latencies for the four groups tested after a single shock. There were no baseline differences between groups $(F<1)$. Rats not preexposed to the shock environment and given only saline became analgesic, with the analgesia gradually dissipating by the end of the $10 \mathrm{~min}$ of testing. The initial analgesia following shock was not attenuated by preexposure to the shock environment. However, the analgesia at 4-8 $\mathrm{min}$ was strongly reduced by such preexposure. Naltrexone blocked the analgesia throughout its entire extent. These impressions were confirmed by a $2 \times 2 \times 5$ repeated measures analysis of variance (ANOVA). The effects of naltrexone versus saline (drug) $[F(1,28)=43.81, p<.0001]$, preexposure to the shock versus nonshock cues (preexposure) $[F(1,28)$ $=6.07, p<.03 \mathrm{~J}$, the interaction between drug and preexposure $[F(1,28)=4.61, p<.05]$, test point (trials) $[F(4,112)=26.53, p<.0001]$, the interaction between drug and trials $[F(4,112)=12.25, p<.0001]$, and the interaction between drug, preexposure, and trials $[F(4,112)$ $=5.78, p<.0005]$ were all reliable. The preexposure $\times$

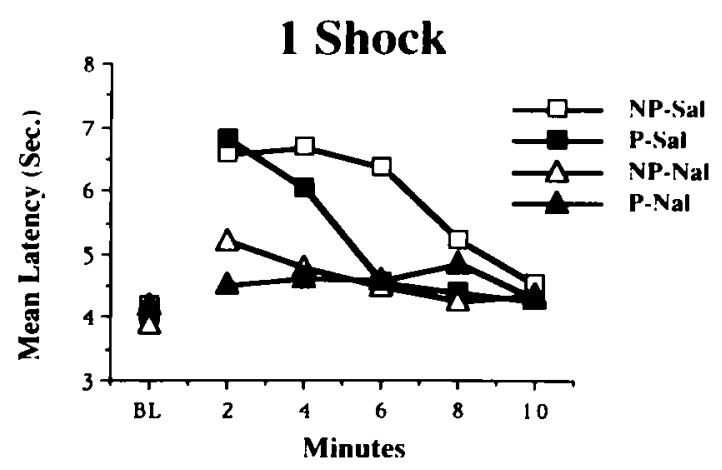

Figure 1. Mean taif-filck latencles at baseline (BL) and 2, 4, 6, 8 , and $10 \mathrm{~min}$ after one shock. Subjects had been preexposed to the shock environment (P) or to a nonshock environment (NP) and had received either naltrexone (Nal) or saline (Sal) before testing. 
trials interaction was not reliable. Subsequent NewmanKeuls comparisons $(p<.05)$ revealed the following pattern at each of the test points. At the 2-min test, the saline groups differed from the naltrexone groups, which did not differ from each other. At the 4-min test, the group that received saline and was preexposed to nonshock cues differed from the naltrexone groups but not from the group that received saline and was preexposed to shock cues. The group that received saline and was preexposed to shock cues did not differ from the naltrexone groups. At the 6-min test, the group that received saline and was preexposed to nonshock cues differed from all of the others, which did not differ among themselves. There were no reliable differences at the 8- and 10-min tests.

Figure 2 shows the results following five shocks. Here, the pattern is quite different. The group preexposed to the nonshock environment and given saline remained analgesic throughout the $10 \mathrm{~min}$ of testing. Neither preexposure to the shock environment nor naltrexone reduced the initial analgesic reaction occurring during the first 4 min postshock, but both reduced the analgesia occurring 6-10 $\mathrm{min}$ postshock. Moreover, the combination of naltrexone and preexposure to shock cues seems slightly more effective than do either separately. A repeated measures ANOVA yielded reliable effects of drug $[F(1,28)=38.34, p<$ $.0001]$, preexposure $[F(1,28)=24.04, p<.0001]$, the drug $\times$ preexposure interaction $[F(1,28)=7.04, p<.02]$, trials $[F(4,112)=53.98, p<.0001]$, the drug $\times$ trials interaction $[F(4,112)=2.81, p<.03]$, the preexposure $\times$ trials interaction $[F(4,112)=5.22, p<.0008]$, and the drug $\times$ preexposure $\times$ trials interaction $[F(4,112)=$ $5.46, p<.0005]$. Subsequent Newman-Keuls comparisons $(p<.05)$ yielded no reliable group differences at the 2- and 4-min tests; a reliable difference between the group that received saline and was preexposed to nonshock cues and the group that received naltrexone and was preexposed to shock cues at the 6-min test; and a reliable difference between the group that received saline and was preexposed to nonshock cues and each of the other three

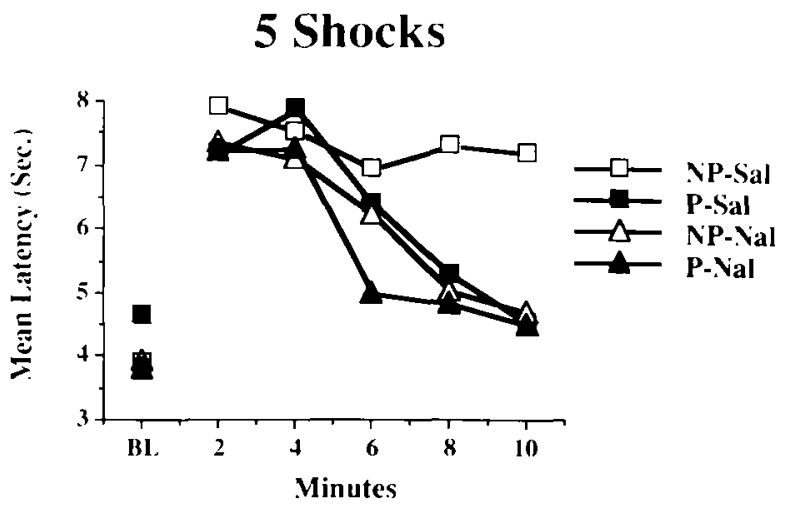

Figure 2. Mean tail-flick latencies at baseline (BL) and 2, 4, 6, 8 , and 10 min after five shocks. Subjects had been preexposed to the shock environment (P) or to a nonshock environment (NP) and had received either naltrexone $(\mathrm{Nal})$ or saline (Sal) before testing.

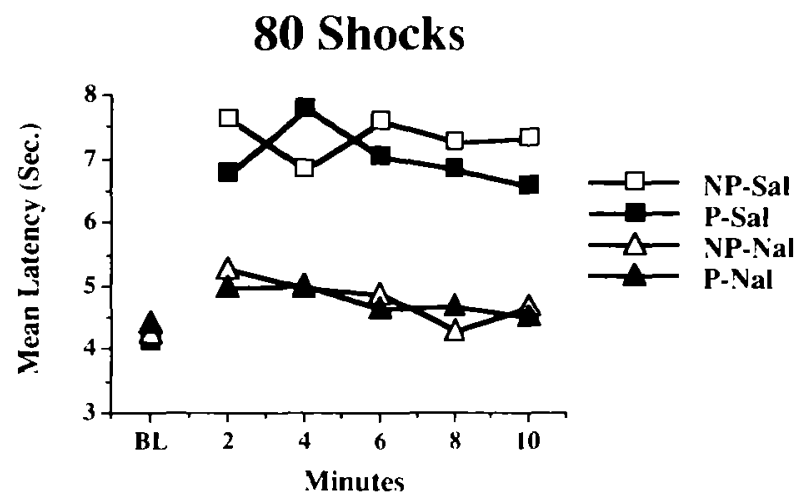

Figure 3. Mean tail-flick latencies at baseline (BL) and 2, 4, 6, 8 , and 10 min after 80 shocks. Subjects had been preexposed to the shock environment (P) or to a nonshock environment (NP) and had received either naltrexone (Nal) or saline (Sal) before testing.

groups at the 8- and 10-min tests. No other differences were reliable.

Figure 3 shows the pattern following 80 shocks. Again, the results were different from those following 1 and 5 shocks. Here, analgesia persisted for the entire 10-min period for the group that received saline and was preexposed to nonshock cues and was blocked for the entire test period by naltrexone. Preexposure to the shock cues had no effect at any test point. A repeated measures ANOVA found a reliable effect of drug $[F(1,28)=$ $150.14, p<.0001]$. Neither the effects of preexposure, the drug $\times$ preexposure interaction, the drug $\times$ trial interaction, nor the 3-way interaction approached significance. The effect of trials approached significance $[F(4,112)=$ $2.24, p<.07]$. Subsequent Newman-Keuls tests $(p<$ $.05)$ found that the naltrexone and saline groups differed from each other, but that the saline and naltrexone groups did not differ among themselves.

These results are straightforward and consistent with the suggestion that there is an early analgesia following small numbers of shocks that is unconditioned in nature, and that this initial analgesia is later replaced by one that is a conditioned reaction. In this experiment, preexposing the rats to the cues that would later be present during shock and subsequent pain testing altered the pattern that follows no preexposure (Maier, 1989) or preexposure to a set of cues different from that accompanying shock and testing. Such preexposure reduced or eliminated the lateappearing analgesia, while leaving the initial analgesia unaffected. Not only was the occurrence of early analgesia unaffected by preexposure to the shock cues, but its opioidnonopioid nature was also unchanged. A single shock was followed by an initial opioid reaction, and 5 shocks by a nonopioid reaction. As before, the second, putatively conditioned analgesia was opioid in both cases. Finally, the analgesia following $\mathbf{8 0}$ shocks again appeared to be a uniform rather than a bipartite sequential reaction and not to be conditioned. It was opioid across its entire extent and unaffected by preexposure to shock cues at any point. 


\section{EXPERIMENT 1B}

It is possible to argue that the failure of preexposure to reduce the analgesia following 80 inescapable shocks does not reflect an insensitivity of this analgesia to latent inhibition, but rather that 80 inescapable shocks produce so much conditioning that latent inhibition is overcome. Experiment 1B was designed to assess this possibility. The strategy was to determine whether preexposure was or was not sufficient to reduce the conditioning that 80 shocks do undoubtedly produce. Remember, the theoretical question is whether the analgesia observed immediately after 80 shocks is an associatively derived response, not whether 80 shocks can condition analgesia that could be observed under different conditions of testing. They undoubtedly can do so. If $\mathbf{8 0}$ shocks are sufficient to overcome latent inhibition, then latent inhibition should not be observed under conditions designed to reveal the conditioned analgesia produced by 80 shocks.

\section{Method}

Subjects. The subjects were 14 rats of the same strain, age, and sex as in Experiment 1A. Housing and general conditions were as above.

Apparatus. The apparatus was the same as that used in Experiment $1 \mathrm{~A}$.

Procedure. After initial handling, the animals were divided into two groups. As in Experiment IA, one was preexposed to the environment in which shocks would later occur, while the other was preexposed to the nonshock environment. Twenty-four hours after the last preexposure, all rats received 80 inescapable tailshocks in the shock environment as in Experiment 1A. Upon completion of the shock series, all animals were immediately removed from the shock tubes and placed in clean tubes in the nonshock environment. They received a tail-flick test every $10 \mathrm{~min}$ for $50 \mathrm{~min}$. A 50 -min session was chosen because pilot work revealed that the analgesia would completely dissipate across this period of time. The subjects were then returned to the shock environment for $10 \mathrm{~min}$ and received a tail-flick test every 2 min.

\section{Results and Discussion}

The tail-flick data are presented in Figure 4. As can be seen in Figure 4A, the group preexposed to the shock environment was no less analgesic during the immediate postshock testing in the nonshock environment than was the group preexposed to the nonshock environment. This replicates the findings of Experiment 1A, with testing here being in the nonshock environment. As is also evident in Figure 4B, analgesia had dissipated by 50 min postshock. The rats did become analgesic when returned to the shock environment. This presumably is a conditioned or associatively derived reaction, and here preexposure exerted an obvious effect. The group preexposed to the shock environment became much less analgesic than did the group preexposed to the nonshock cues. A repeated measures ANOVA applied to the tail flicks measured upon return to the shock environment yielded reliable effects of preexposure $[F(1,12)=11.77, p<.005]$ and trials $[F(4,48)$ $=6.86, p<.0002]$. The preexposure $\times$ trials interaction was not reliable.

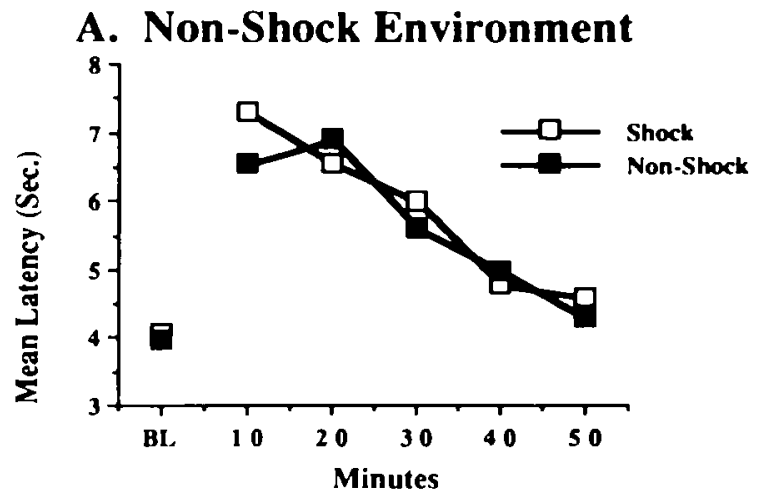

\section{B. Shock Environment}

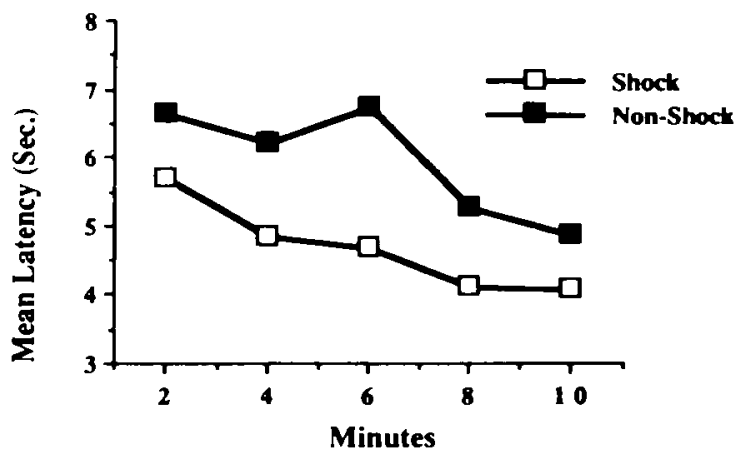

Figure 4. (A) Mean tall-nick latencies at baseline (BL) and 10, $20,30,40$, and $50 \mathrm{~min}$ after 80 shocks followed by removal to the nonshock environment. Subjects had been preexposed to the shock or nonshock environment. (B) Mean tall-nick latencies 2, 4, 6, 8, and $10 \mathrm{~min}$ after return to the shock environment. Subjects had been preexposed to the shock or nonshock environment.

These data make it difficult to argue that the failure of preexposure to alter the analgesia immediately following 80 inescapable shocks is attributable to a preexposure insufficient to reduce conditioning produced by so many shocks. The preexposure was indeed sufficient to reduce the amount of conditioning when test conditions that assessed conditioned responding were used. This further supports the argument that the analgesia that occurs immediately following 80 inescapable shocks is simply not a reaction deriving from Pavlovian associative processes.

\section{EXPERIMENT 2}

One of the original experiments (Maier, 1989, Experiment 3) suggesting that the analgesia that occurs 2-4 min after a shock is unconditioned while the subsequent analgesia that occurs is conditioned was one in which the animals were removed from the shock tubes for pain testing. Rats received either 1,5 , or 80 tailshocks and were immediately removed from the tubes after their last shock. They were quickly taken to a different room in which animals had never been shocked and in which the light- 
ing and noise conditions were quite different from those in the shock room. There, the animals were placed into clean tubes and received tail-flick testing across the usual 10-min interval. The logic was that if the early analgesia is an unconditioned reaction to the shock, then it ought to be triggered by shock and simply run its course. Thus, this unconditioned analgesia should have been observable in the new tubes in the new room. If the second analgesia is in fact a conditioned reaction elicited by the cues that had been paired with shock, then this reaction might be expected to be weak or absent during testing, since many of the cues that had been paired with shock were not present during testing. After 80 shocks, the pattern was expected to be quite different. If the analgesia after 80 shocks is a reaction that is triggered by having learned that the shock is uncontrollable and that runs its course once initiated, then the reaction might be expected to persist in an undiminished fashion in the new environment.

The results followed the above pattern. The animals that had been given 1 shock were only briefly ( $2 \mathrm{~min}$ ) analgesic when tested in the new environment. The rats that had received 5 shocks were analgesic for 4-6 $\mathrm{min}$, but the second analgesia did not appear. It was known that it was the second analgesia that did not occur in the new environment because naltrexone had no effect on the analgesia that did occur. Recall that naltrexone has no effect on the initial analgesia after 5 shocks, but blocks the analgesia that occurs at 6-10 min after shock if the animals are allowed to remain in the shock environment. Thus, the fact that naltrexone had no effect on the analgesia in the new environment suggested that the analgesia in the new environment was of the type that follows immediately after 5 shocks. The rats that had received 80 shocks remained analgesic throughout the 10 -min test, although the analgesia seemed somewhat smaller than that which occurs if the subjects are not removed from the shock tubes.

The fact that the analgesia occurred 4-10 min after a small number of shocks if the subjects remained in the presence of shock cues, but did not occur if they were removed from the shock cues, is consistent with the possibility that the analgesia that occurs at the 4-10-min delay is dependent on the presence of stimuli that had been paired with shock and is therefore associatively aroused. However, there are uncertainties with regard to this conclusion. For example, it is possible that the analgesia at these time points is a nonassociatively derived reaction that is induced by the initial analgesic reaction as a consequence of that reaction. Alternatively, the shock itself may produce a biphasic reaction with one type followed by the other type (Grau, 1987b). Perhaps removing the subjects from the shock environment and placing them in a new environment led to the appearance of only the first analgesia not because the second reaction required shock cues, but because the removal and placement in the new environment disrupted the sequential processes.

The purpose of Experiment 2 was to further explore the "removal" paradigm and the possible associative nature of the analgesia after small numbers of shocks.
Several predictions follow from the argument that the analgesia present 4-10 min after shock in the shock environment is elicited by the shock cues because the cues have been paired with shock. The most obvious prediction is that rats shocked in one environment and removed and tested in a different environment, as in Maier (1989), would become analgesic again if returned to the shock environment after removal from the nonshock cues. This follows because the shock cues should not lose their conditioned properties while the rat is in the nonshock environment, presuming that the cues are sufficiently distinct to minimize generalization. Along the same lines, it would also be expected that the associative strength of the shock cues could be extinguished so that returning the subjects to the shock environment would now fail to produce appreciable analgesia.

\section{Method}

Subjects. The subjects were 64 male Sprague-Dawley rats, 80-90 days old at the time of testing. The rats were individually housed and maintained on a 12:12-h light:dark cycle, with testing typically occurring in the first few hours of the light part of the cycle.

Apparatus. The apparatus was identical to that used in Experiment 1.

Procedure. All subjects were first accustomed to handling. On the experimental day, half received a $7 \mathrm{mg} / \mathrm{kg}$ s.c. injection of naltrexone hydrochloride $(\mathrm{Nal})$, while the other half received equivalent volumes of the saline vehicle (Sal). Twenty minutes later, all rats received baseline tail-flick testing in the shock room as in Experiment 1 , followed by five shocks in the shock room with cues as in Experiment 1. The one-shock condition was not examined in this experiment because the relatively weak second analgesic reaction produced by a single shock would have made any results in the present experiment difficult to interpret. Eight $\mathrm{Nal}$ and $8 \mathrm{Sal}$ subjects then received 10 min of tail-flick testing (tests $2,4,6,8$, and $10 \mathrm{~min}$ after the last of the five shocks) without being removed from the shock tubes or shock room. This is the normal, baseline procedure from which deviations will be assessed, so the groups are called $\mathrm{Nal}$-normal and Sal-normal.

A second set of $\mathrm{Nal}$ and $\mathrm{Sal}$ animals was removed from the shock tubes immediately after the fifth shock, taken to the nonshock room, and placed in clean tubes. After $10 \mathrm{~min}$ in the nonshock environment (the condition during which the second analgesia did not occur in Maier, 1989, and by the end of which there was no analgesia at all), these subjects were returned to the shock room, placed in the same "dirty tubes" out of which they had been taken, and tail-flick tested for $10 \mathrm{~min}$. Tail-flick testing was not conducted during the $10 \mathrm{~min}$ in the nonshock environment because the logic of the experiment demands comparison of tail-flick latencies in the shock room for the various groups, so the occurrence of prior testing in the nonshock room would have been a confounding factor. These groups are designated Nal-nonshock 10 and Sal-nonshock 10. A separate group of 8 rats was employed to assure that the analgesia does in fact dissipate by the end of the $10 \mathrm{~min}$ in the nonshock environment, as in Maier (1989). There was no analgesia at all after $10 \mathrm{~min}$ in the nonshock environment $(F<1.0)$.

A third set of $\mathrm{Nal}$ and $\mathrm{Sal}$ rats received five shocks, as above. They then remained in the shock tubes for $20 \mathrm{~min}$ with no further shock or tail-flick testing. Pilot work revealed that analgesia is no longer present after $20 \mathrm{~min}$ in the tube, presumably because it has extinguished. The animals in this group were then removed from the shock tubes and shock room and taken to the nonshock room, placed in clean tubes, and allowed to remain there for $10 \mathrm{~min}$. They were then removed from the clean tubes and nonshock room, 
returned to the dirty tubes in the shock room, and given $10 \mathrm{~min}$ of tail-flick testing as in the above groups. These two groups are designated Nal-shock and Sal-shock.

Thirty minutes elapsed between the last shock and return to the shock environment and testing in the Nal-shock and Sal-shock groups. Thus, any failure of analgesia to occur during testing could be attributed to the 30 -min interval, rather than to any putative extinction that occurred in the $20 \mathrm{~min}$ in the shock environment. Thus, a final set of $\mathrm{Nal}$ and $\mathrm{Sal}$ animals received five shocks, were then removed from the shock tubes and shock room, and placed in clean tubes in the nonshock room for $30 \mathrm{~min}$. They were then removed, returned to the dirty tubes in the shock room, and given $10 \mathrm{~min}$ of tail-flick testing as in all of the other groups. These groups are labeled $\mathrm{Nal}$-nonshock 30 and Sal-nonshock 30.

In summary, all groups received five shocks and eventual testing in the shock environment across a 10-min period. They differed with regard to their treatment between the last of the five shocks and the test. One group was simply tested without any intervening treatment; one was taken to a nonshock environment for $10 \mathrm{~min}$; one was taken to a nonshock environment for $30 \mathrm{~min}$; and one remained in the shock environment for $20 \mathrm{~min}$, then was placed in the nonshock environment for $10 \mathrm{~min}$

\section{Results and Discussion}

For ease of inspection, the results are depicted separately for the $\mathrm{Nal}$ and $\mathrm{Sal}$ groups. The tail-flick latencies for the Sal groups are shown in Figure 5A. As can be seen, group Sal-normal had the pattern of analgesia expected after five shocks with immediate testing in the shock environment. The animals were strongly analgesic and remained so for the $10 \mathrm{~min}$ of testing, with some diminution by the 10-min point. Group Sal-nonshock 10 is the group assessed by Maier (1989) and in a separate experiment here in which analgesia was no longer present by the end of the $10 \mathrm{~min}$ in the nonshock environment. As is evident, returning them to the shock environment led to a new analgesic reaction, somewhat smaller than in Sal-normal, that became substantially smaller by the end of testing. Group Sal-nonshock 30 also became analgesic when returned to the shock environment after $30 \mathrm{~min}$ confinement in the nonshock environment, and their level of analgesia was indistinguishable from that of Sal-nonshock 10. Finally, the Sal-shock group showed very little analgesia upon return to the shock environment.

Figure 5B shows tail-flick latencies for the Nal groups. Group Nal-normal showed the usual pattern seen in prior research. Analgesia was not affected during the first $4 \mathrm{~min}$ postshock but was reduced thereafter. None of the other groups showed any analgesia.

These conclusions were confirmed by a 2 (drug) $\times 4$ (treatment before testing) $\times 5$ (trials) repeated measures ANOVA. The effects of drug $[F(1,58)=127.68, p<$ $.0001]$, treatment before testing $[F(3,58)=83.43, p<$ $.0001]$, the interaction between drug and treatment before testing $[F(3,58)=17.49, p<.0001]$, trials $[F(4,232)=$ $8.64, p<.001]$, the interaction between trials and treatment before testing $[F(12,232)=4.56, p<.0001]$, and the interaction between trials, drug, and treatment before testing $[F(12,232)=4.38, p<.0001]$ were all reliable. The interaction between drug and trials was not reliable. Subsequent Newman-Keuls tests $(p<.05)$ revealed the
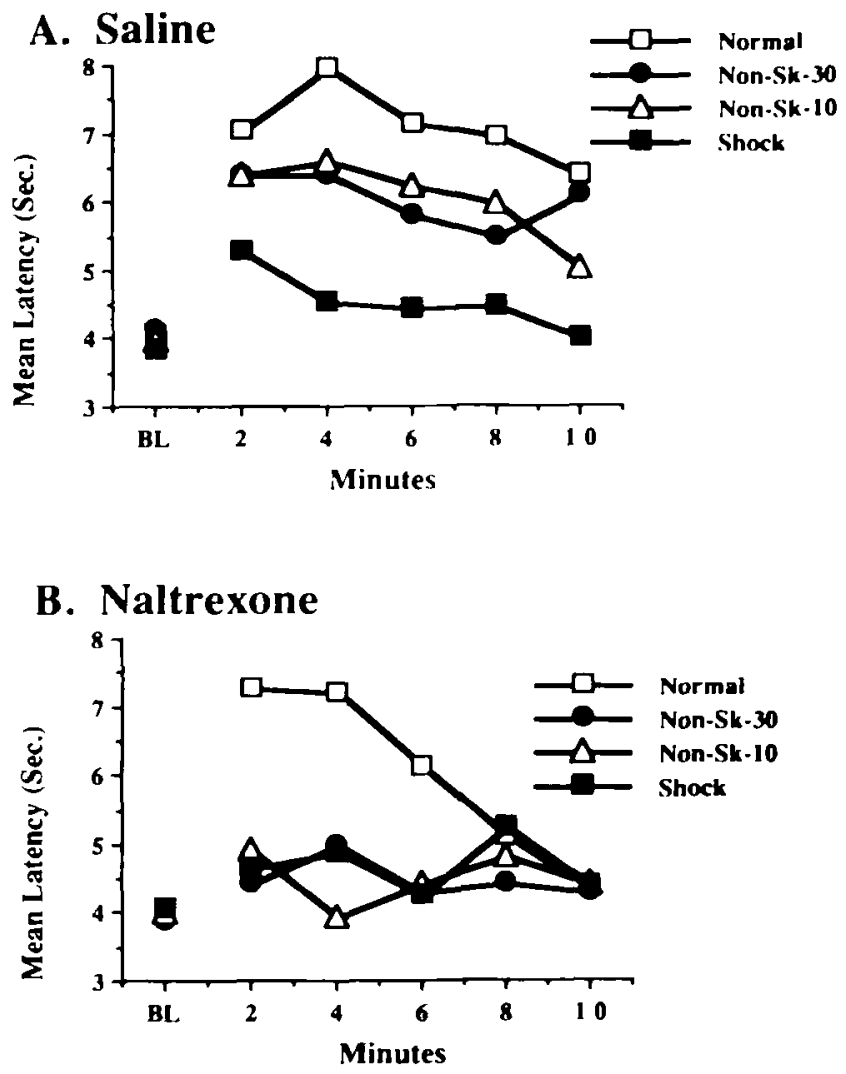

Figure 5. (A) Mean tail-nick latencies at baseline (BL) and 2, 4, 6,8 , and $10 \mathrm{~min}$ after the start of testing. All subjects had received saline before five shocks and were tested in the shock environment. One group was tested immediately after the last shock (Normal); one was removed and pleced in a nonbock environment for 10 min before testing (Non-SS-10); one was removed to a non hock environment for 30 min (Non-Sk-30); and ane remained in the shock environment for $20 \mathrm{~min}$, was removed for $10 \mathrm{~min}$, and then was returned to the shock environment for testing (Shock). (B) Mean tail-fick latencies at baseline (BL) and 2, 4, 6, 8, and 10 min anter the start of testing. All subjects had recelved naltrexone before five shocks and were tested in the shock environment. One group was tested immediately after the last shock (Normal); one was removed and placed in a nonshock environment for $10 \mathrm{~min}$ before testing (Non-S1-10); one was removed to a nonchock environment for $30 \mathrm{~min}$ (Non-Si-30); and one remained in the shock environment for $20 \mathrm{~min}$, was removed for $10 \mathrm{~min}$, and then was returned to the shock environment for testing (Shock).

following pattern at each test point: 2 min: Sal-normal, $\mathrm{Nal}$-normal, Sal-nonshock 10, and Sal-nonshock 30 differed from the other four groups but did not differ among themselves. The remaining four groups did not differ among themselves; $4 \mathrm{~min}$ : The pattern was the same as at 2 min with the exception that Sal-normal differed from Sal-nonshock 30; 6 min: Sal-normal differed from all of the other groups. Sal-nonshock 10, Sal-nonshock 30, and $\mathrm{Nal}$-normal differed from the remaining groups, and the remaining groups did not differ from each other; $8 \mathrm{~min}$ : The pattern was the same as that at $6 \mathrm{~min} ; 10 \mathrm{~min}$ : Salnormal differed from all of the other groups except Sal- 
nonshock 30. Sal-nonshock 30 differed from all others except Sal-nonshock 10. None of the remaining groups differed from each other.

In summary, the subjects did become analgesic again upon return to the shock environment after 10 or $30 \mathrm{~min}$ in a nonshock environment. Even though analgesia was no longer present after $10 \mathrm{~min}$ in the presence of nonshock cues, potent analgesia was produced upon return to the presence of shock cues. This analgesia did not $\alpha$ cur if naltrexone was administered before the experimental treatment, in keeping with the general finding that conditioned analgesia is opioid in nature using the present parameters and procedures. The analgesia did not occur if the subjects were kept in the presence of the shock cues without the occurrence of shock before removal to the nonshock environment, further supporting the proposition that this analgesia that occurred upon return to the shock environment is a conditioned reaction. This manipulation should have extinguished conditioned reactions, and this was the result obtained.

It might seem that the presence of "shock odors" in the tubes to which the animals were returned for testing presents a difficulty for these conclusions. These odors were present during the initial shock treatment and were allowed to remain present since they were therefore part of the shock context. However, "stress odors" have been reported to produce analgesia as an unconditioned reaction (Fanselow, 1985). Thus, it is possible that the analgesia observed upon return to the shock environment is an unconditioned reaction to stress odors rather than a conditioned reaction.

However, this factor cannot account for the difference in the magnitude of the analgesic reactions between the groups. Conditioned analgesia is inferred from the different levels of analgesia among groups Sal-nonshock 30, Sal-nonshock 10, and Sal-shock. All three groups were returned to the tubes with stress odors present, with return occurring at the same amount of time postshock. Thus, any unconditioned reactions to odors should have been equated among the three groups, yet they reacted with different levels of analgesia. The amount of analgesia observed in the Sal-shock group represents the maximal amount that could be attributed to an unconditioned reaction to odors, and it is quite small. It is too small to account for the analgesia in the other groups, so the conclusions concerning conditioned analgesia are secure. Indeed, this slight analgesia might not even reflect unconditioned reactions to odors, but might instead be wholly or partly a reflection of spontaneous recovery of extinguished conditioned analgesia. This suggestion is made because the baseline tail flicks were measured on shock days in the shock room in dirty tubes containing stress odors, and thus should already include any analgesia produced by odors.

\section{GENERAL DISCUSSION}

The purpose of the reported experiments was to explore the associative nature of the analgesia that follows ex- posure to electric tailshocks. A number of quite different views have been proposed. Perhaps the simplest is that shock produces an analgesic reaction as a direct unconditioned reaction to shock mediated by interaction between the sensory input and circuitry in the brainstem and/or spinal cord (Maier, 1986; Terman et al., 1984). For this view, associative processes and psychological processing of the stimulus more generally play little if any role. Supporting this view are experiments showing that decerebration and pentobarbital anesthesia do not prevent shock from producing analgesia (Maier, 1989; Terman et al., 1984; Watkins et al., 1982). It has been clear that the nature of the analgesia changes if the shocks are inescapable and a large number occur. Thus, it has been additionally argued that the unconditioned reaction eventually habituates and is replaced by a reaction dependent on learning that the inescapable shocks are indeed inescapable (Maier, 1986).

Fanselow (1986) has proposed a different position. He argues that signals for danger activate a defensive motivational system and that analgesia is part of this system. Here, explicit signals for danger and not painful events themselves produce analgesia. The signals can be either innate or acquired. In the case of shock, the signal is an acquired or conditioned signal. Thus, for this view the analgesia produced by shock is always an associatively derived reaction. Indeed, Fanselow has provided a variety of experiments attesting to the conditioned nature of the analgesia that follows a small number of shocks (Fanselow, 1984). Fanselow is explicit that his position is restricted to the outcome of procedures using small numbers of shocks (Fanselow, 1986).

Grau (1987a, 1987b) has also emphasized the role of associative processes. Here, analgesia is placed within the context of Wagner's SOP model of associative processes (Wagner, 1981). This position is complex and can allow for a variety of patterns of data. It allows for analgesia that is both nonassociatively and associatively derived. The simple presentation of an event can enter it into the $A 1$ state (argued to produce nonopioid analgesia), and a cue that has been paired with an event can enter it into the A2 state (argued to produce opioid analgesia). This position does, however, require "higher level processing" of the event. Even here, however, Grau (1987a) has argued that events such as shock can produce analgesia without entering into working memory at all and without higher level processing if they are very intense. Finally, Grau (1987b) has argued that the mechanisms responsible for analgesia do not become fundamentally different after a large number of inescapable shocks. It is the representation of shock in the $\mathrm{A} 2$ state that produces analgesia, with the analgesia being potentiated by pituitary-adrenal hormones activated by the prolonged shock exposure. Grau's position will not be discussed further because the implications of the present data for his model are not clear.

Not only have these different positions been proposed, but evidence supporting each has been provided. Maier $(1989,1990)$ has argued that many of the seemingly discrepant findings can be reconciled by a careful analysis of the way in which each of the groups has conducted their 
experiments. The groups that have obtained evidence for unconditioned effects mediated at brainstem and/or spinal sites (Liebeskind/Terman; Maier; Mayer/Watkins) have generally either tested their subjects very soon after the last shock and/or have removed their subjects from the shock environment for pain testing. The groups that have obtained evidence for a crucial role for higher level processing and associative processes (Fanselow; Grau) have generally either tested for pain in the shock environment itself, have summed pain-test data over a long postshock period, or both. Obviously, the first set of conditions would maximize measurement of any unconditioned effects that might be produced by shock, while the second set would maximize measurement of any conditioned or associative effects.

These considerations led Maier $(1989,1990)$ to conduct a series of experiments that manipulated these variables. The outcomes suggested that tailshock leads to both unconditioned and conditioned effects, with the conditions of testing determining which is observed. After a small number of shocks, the last shock in the series seemed to be followed by an analgesic reaction that was observable regardless of whether testing occurred in the shock environment or in a different environment. The opioid/ nonopioid nature of this reaction was determined by the number of shocks. This first reaction dissipated and was replaced by a different reaction, but only if the animals had not been removed from the shock environment for testing. This reaction was always opioid. Maier argued that the first reaction represented an unconditioned reaction and the second a conditioned response because the first reaction was observable in anesthetized animals and the second was dependent on an awake state and was prevented by removal from the shock environment. Because the entire analgesic reaction observed after 80 inescapable tailshocks depended on the awake state and was unaffected by removal from the shock environment, Maier (1989) argued that it was neither an unconditioned nor a conditioned response, but rather a reaction set in motion by having learned that the shocks are inescapable.

Although the data reported by Maier (1989) are consistent with his arguments, they are hardly conclusive. Thus, the present experiments were conducted to further test these hypotheses. The core of the argument was that Pavlovian associative processes are not involved in generating the analgesic reaction that occurs after large numbers of inescapable shocks or 0-4 min after small numbers of shocks. Pavlovian associative processes were seen as generating only the analgesic reaction that begins 2-4 min after the last of a small number of shocks. This would suggest that manipulations that modulate the level of Pavlovian conditioning should affect only the analgesia beginning $4 \mathrm{~min}$ after the last of a small number of shocks. Preexposure to the to-be-conditioned stimulus (latent inhibition) is known to be a potent modulator of Pavlovian conditioning. In Experiment 1, subjects were preexposed to either the environment in which shocks later were administered or to a distinctively different environ- ment. Preexposure to the environment in which shock was later administered had no effect on the analgesic pattern observed after 80 inescapable shocks, the analgesia 2 and 4 min after 1 shock, or the analgesia 2,4 , and 6 min after 5 shocks. However, preexposure did reduce or eliminate the analgesia subsequent to the 4-min test point after 1 shock and the 6-min test point after 5 shocks. As in previous work, the analgesia soon after shock was either reversed by naltrexone or insensitive to naltrexone, depending on the number of shocks. Also as before, the second reaction was sensitive to naltrexone, as was the analgesia following 80 inescapable shocks. This again confirms the existence of a biphasic analgesic pattern after small numbers of shocks, with the first reaction being insensitive to variables that alter associative conditioning processes and the second being sensitive to such variables. This also further confirms the insensitivity of the analgesia to conditioning variables following 80 inescapable shocks.

Experiment 2 added evidence indicating that the shock environment used here does produce hypoalgesias as a reaction to the stimuli of the environment rather than only as a reaction to the shock. Subjects who were returned to the shock environment after the postshock analgesia had been allowed to dissipate in a nonshock location became analgesic again. Moreover, the analgesia did not then have a biphasic nature and was opioid throughout its entire event. Finally, the ability of the shock environment to elicit analgesia upon reexposure of the subjects to the environment was reduced if the subjects were kept in the shock environment without further exposure to shock. This is what would be expected if the shock environment elicited analgesia as a conditioned response.

The present experiments add to prior work in strongly suggesting that shock does produce analgesia as an unconditioned reaction. There is a decrease in pain sensitivity/ reactivity immediately following shock that is not reduced by prior exposure to the stimuli present during shock (Experiment 1), that is not reduced by manipulations designed to reduce fear (Maier, 1990), that is not disrupted by removal of the subject from the shock environment (Maier, 1989), that does not occur again if the subjects are reexposed to the shock environment after the analgesia has been permitted to dissipate in a nonshock environment (Experiment 2), and that does not require the awake state to occur (Maier, 1989). Thus, any position that argues that analgesic processes can only be triggered by signals for danger will have to be modified.

It might be noted that Fanselow (1991) has recently modified his model in this manner to incorporate an unconditioned analgesia. What is critical for Fanselow's model is that signals for danger produce analgesia, and the data reported here strongly support this assertion. It is much less critical that this be the only access to the circuitry that mediates analgesia, and Fanselow has added this access. Moreover, it is quite likely that the unconditioned and conditioned hypoalgesias are mediated by different physiological substrates. The data reported here demonstrate that conditioned analgesia involves an opi- 
oid mechanism, whereas the unconditioned analgesia after five shocks does not. Data reported elsewhere (Maier, 1989) show that the unconditioned analgesia following more than five shocks is also insensitive to naltrexone. Unpublished data from our laboratory indicate that it is only after one and two tailshocks that the unconditioned analgesia is opioid. Thus, under most conditions unconditioned analgesia is nonopioid, whereas conditioned analgesia is opioid. It can be further noted that even after one and two shocks the opioid nature of unconditioned and conditioned analgesia might be quite different. There are a number of different endogenous opioid peptides, opioid receptors, and central nervous system structures where opioids play a role in pain modulation. It may well be that conditioned and unconditioned opioid hypoalgesias utilize fundamentally different opioid mechanisms. Thus, it would be an easy matter for Fanselow to restrict the link between the defensive motivational system and analgesia systems to a particular system. In this regard, Fanselow, Calcagnetti, and Helmstetter (1989) have argued that conditioned analgesia is mediated by mu receptors in the brain, rather than by delta or kappa opiate receptors.

The present experiments also have implications for the analgesia that occurs following larger numbers of inescapable shocks. Here, the issue is whether the opioid analgesia produced by 80 inescapable shocks is fundamentally different from the unconditioned opioid analgesia following small numbers of shocks and conditioned analgesia. The present data are consistent with prior findings suggesting that the analgesia produced by 80 inescapable shocks differs substantially from the other opioid hypoalgesias (Maier, 1989). Here, we add the finding that prior exposure to the environment in which shock occurs reduces or prevents conditioned analgesia, but has no detectable effect on the analgesia that follows 80 inescapable shocks. This again supports the argument that the reaction is not a conditioned reaction in any ordinary sense. It is affected neither by the removal of cues that have been paired with shock (Maier, 1989), nor by preexposure to those cues. This is perhaps not surprising since the only real reason to believe that the hypoalgesias are similar is that they are all reversed by high doses of systemically administered naltrexone. However, the involvement of opioid peptides in pain modulation is quite complex, with multiple opioid systems in both brain and spinal cord with different receptor types playing roles. Systemic administration of naltrexone would have an impact on both brain and spinal receptors, and additionally, the doses typically used are not selective for a given receptor subtype. It is thus quite possible that the various types of opioid hypoalgesias described here do not employ the same opioid circuits and differ even with regard to the details of their opioid nature.

\section{REFERENCES}

FANSELOW, M. S. (1984). Shock-induced analgesia on the formalin test: Effects of shock severity, naloxone, hypophysectomy, and associative variables. Behavioral Neuroscience, 98, 79-95.

FANSELow, M. S. (1985). Odors released by stressed rats produce opioid analgesia in unstressed rats. Behavioral Neuroscience, 99, 589-592.

FANSELOW, M. S. (1986). Conditioned fear-induced opiate analgesia: A competing motivational state theory of stress analgesia. Annals of the New York Academy of Sciences, 467, 40-54.

FANSELOW, M.S. (1991). The midbrain periaqueductal gray as a coordinator of action in response to fear and anxiety. In A. Depaulis \& R. Bandler (Eds.), The midbrain PAG (pp. 151-173). New York: Plenum.

Fanselow, M. S., Calcagnetti, D. J., Helmstetter, F. J. (1989). The role of mu and kappa opioid receptors in conditional-fear induced analgesia: The antagonistic actions of Nor-binaltorphimine and the cyclic somatostatin octapeptide $\mathrm{Cys}^{2} \mathrm{Tyr}^{3} \mathrm{Om}^{3} \mathrm{Pen}^{7}$-Amide. Journal of Pharmacology and Experimental Therapeutics, 250, 825-830.

GrAU, J. W. (1987a). The activation of the opioid and nonopioid analgesic systems: Evidence for a memory hypothesis and against the coulometric hypothesis. Joumal of Experimental Psychology: Animal Behavior Processes, 13, 215-225.

GraU, J. W. (1987b). The central representation of an aversive event maintains the opioid and nonopioid forms of analgesia. Behavioral Neuroscience, 101, 272-288.

Hayes, R. L., Bennett, G. J., Newlon, P., \& Mayer, D. J. (1978). Behavioral and physiological studies of non-narcotic analgesia in the rat elicited by certain environmental stimuli. Brain Research, 155 , $69-90$

Klein, M. V., LovaAs, K. M., Terman, G. W., \& Liebeskind, J. C (1983). The effects of decerebration and spinal transection on three discrete forms of stress-induced analgesia. Society of Neuroscience Abstracts, 9, 795

Lubow, R. E., Moore, A. U. (1959). Latent inhibition: The effect of nonreinforced preexposure to the conditioned stimulus. Joumal of Comparative \& Physiological Psychology, 52, 415-419.

Maclennan, A. J., Jackson, R. L., Maier, S. F. (1980). Conditioned analgesia in the rat. Bulletin of the Psychonomic Society, 15. 387-390.

MAIER, S. F. (1986). Stressor controllability and stress-induced analgesia. Annals of the New York Academy of Sciences, 467, 55-72.

MAIER, S. F. (1989). Determinants of the nature of environmentallyinduced analgesia. Behavioral Neuroscience, 103, 131-143.

MAIER, S. F. (1990). Diazepam modulation of stress-induced analgesia depends upon the type of analgesia. Behavioral Neuroscience, 104, 339-347.

Terman, G. W., Shavit, Y., Lewis, J. W., Cannon, J. T., \& LIEBESKIND, J. C. (1984). Intrinsic mechanisms of pain inhibition: Activation by stress. Science, 226, 1270-1277.

WAGNER, A. R. (1981). SOP: A model of automatic memory processing in animal behavior. In N. E. Spear \& R. R. Miller (Eds.), Information processing in animals: Memory mechanisms (pp. 5-47). Hillsdale, NJ: Erlbaum.

Watkins, L. R., Cobelli, D. A., \& Mayer, D. J. (1982), Classical conditioning of front paw and hind paw footshock induced analgesia (FSIA): Naloxone reversibility and descending pathways. Brain Research, 243, 119-132.

(Manuscript received April 12, 1991; revision accepted for publication August 5, 1991.) 